\title{
Estado y rasgo de ansiedad en escolares y adolescentes colombianos con y sin desórdenes gastrointestinales funcionales
}

\section{Anxiety status and trait in Colombian schoolchildren and adolescents with and without functional gastrointestinal disorders}

Carlos Alberto Velasco-Benítez, MD, ${ }^{1 *}$ Carmen Rossy Ramírez-Hernández, MD, ${ }^{2}$ Daniela Alejandra Velasco-Suárez. ${ }^{3}$

Pediatra, gastroenterólogo y nutriólogo, profesor titular del Departamento de Pediatría, Universidad del Valle. Cali, Colombia. ORCID: https://orcid.org/0000-0002-4062-5326 Pediatra. Hospital Regional María Inmaculada, Florencia, Colombia. ORCID: http://orcid. org/0000-0003-1180-9086

Estudiante de Medicina. Pontificia Universidad Javeriana. Cali, Colombia. ORCID: https://orcid. org/0000-0002-6430-0905

*Correspondencia:

Carlos Alberto Velasco-Benítez, MD, carlos.velasco@correounivalle.edu.co

Fecha recibido: $\quad 17 / 06 / 19$

Fecha aceptado: 05/08/19

\begin{abstract}
Resumen
Introducción: la fisiopatología de los desórdenes gastrointestinales funcionales (DGF) incluye un eje intestino-cerebro alterado. Objetivo: determinar la prevalencia y posibles asociaciones de ansiedad en niños con DGF. Metodología: estudio de prevalencia realizado en ciudades colombianas en niños entre 8 y 18 años a quienes se les identificaron DGF según los Criterios de Roma III y ansiedad por medio del State Trait Anxiety Inventory for Children. Se incluyeron variables sociodemográficas y familiares. El análisis estadístico incluyó medidas de tendencia central, análisis uni- y multivariados, y regresión logística, teniendo en cuenta una $p<$ 0,05 como significativa. Resultados: se incluyeron 1496 niños, $12,7 \pm 2,1$ años, 50,5 \% niñas, 79,9 \% con ansiedad transitoria y $51,5 \%$ con tendencia a experimentar estados de ansiedad. Hubo predominio para el estado-ansiedad en adolescentes masculinos y para el rasgo-ansiedad en adolescentes femeninas con algún DGF. Los posibles factores de riesgo fueron la edad y el sexo para estado-ansiedad, y la edad, el sexo y los DGF para el rasgo-ansiedad. Conclusiones: cerca de la mitad de los niños tuvo tendencia a presentar estados de ansiedad, con predominio de las adolescentes femeninas y con factores de riesgo como la edad, el sexo y tener algún DGF.
\end{abstract}

Palabras clave

Ansiedad, enfermedades del sistema digestivo, niños.

\begin{abstract}
Introduction: The pathophysiology of functional gastrointestinal disorders includes alteration of the gut-brain axis. Objective: This study measures prevalence and of functional gastrointestinal disorders and discusses possible associations with anxiety in children with these conditions. Methodology: This is a prevalence study of children between 8 and 18 years of age diagnosed with functional gastrointestinal disorders as defined by the Rome III Criteria and anxiety as defined by the State-Trait Anxiety Inventory for Children in several Colombian cities. Sociodemographic and family variables were included. Statistical analyses included measures of central tendency, univariate and multivariate analysis, and logistic regression, with $p<0.05$ established as significant. Results: The study included 1,496 children of whom $50.5 \%$ were girls. The boys average age was $12.7 \pm 2.1$ years, $79.9 \%$ had suffered transitory anxiety, and $51.5 \%$ had tendencies to experience states of anxiety. State/anxiety predominated in male adolescents while trait/anxiety predominated in female adolescents with functional gastrointestinal disorders. Possible risk factors for state/anxiety were age and sex. Possible risk factors for trait/anxiety were age, sex, and functional gastrointestinal disorders. Conclusions: About half of the children tended to states of anxiety. Female adolescents predominated with risk factors of age, sex, and some functional gastrointestinal disorders.
\end{abstract}

Keywords

Anxiety, diseases of the digestive system, children. 


\section{INTRODUCCIÓN}

Los desórdenes gastrointestinales funcionales (DGF) en escolares y adolescentes se definen como una combinación diversa y variable de síntomas gastrointestinales recurrentes o crónicos que, luego de una adecuada evaluación médica, no son atribuibles a otras condiciones médicas (1). Los criterios de Roma proporcionan pautas basadas en los síntomas mediante las cuales se pueden diagnosticar los DGF en escolares y adolescentes $(1,2)$. Los criterios de Roma III de 2006 enfatizan que "no debe existir evidencia de enfermedad orgánica”, lo que lleva a que su diagnóstico se base en exámenes paraclínicos (2). En los criterios de Roma IV de 2016 se eliminó la frase «que no haya evidencia de un proceso inflamatorio, anatómico, metabólico o neoplásico que explique los síntomas del niño»; y fue sustituida por la frase "después de una evaluación médica apropiada, los síntomas no pueden atribuirse a otra condición médica"; cambio que permite realizar paraclínicos selectivos o no ser necesariamente requeridos para diagnosticar los DGF (1). Estos últimos tienen una prevalencia a nivel mundial entre el $10,0 \%$ y $30,0 \%(3,4)$.

Los Criterios de Roma IV proponen unas características fisiopatológicas para los DGF en las que el eje intestinocerebro está alterado, entre otros, por la gravedad del dolor (hipersensibilidad visceral) y el estrés psicosocial (ansiedad, depresión, impulsividad e ira) (1).

La escala del State Trait Anxiety Inventory for Children 1 (STAIC-1) (estado-ansiedad) es designada para medir los estados de ansiedad transitoria (situaciones de estrés) y la escala del STAIC-2 (rasgo-ansiedad) mide la tendencia relativamente estable a experimentar estados de ansiedad (tendencias neuróticas) (5).

Algunos estudios en niños han demostrado que existe comorbilidad entre el dolor abdominal recurrente (DAR) y la presencia de ansiedad entre un 67,7\% (6)-79\% (7) y un $81 \%$ (8)-81,6\% (9). Teniendo en cuenta que el DAR y la ansiedad están estrechamente relacionados, un mayor entendimiento sobre ambos desórdenes es importante para comprender la fisiopatología del DAR y así proponer medidas preventivas y terapéuticas de este trastorno digestivo funcional.

El objetivo del presente artículo es determinar la prevalencia y posibles factores de riesgo de ansiedad en escolares y adolescentes colombianos con DGF.

\section{METODOLOGÍA}

Estudio observacional descriptivo tipo corte transversal realizado en 4 colegios públicos del tercero a once grado de ciudades colombianas (La Unión, Nariño; Cartagena, Bolívar; Florencia, Caquetá; y Santander de Quilichao, Cauca) en escolares y adolescentes de 8 a 18 años de edad entre el 24 de febrero y el 18 de noviembre de 2014, por lo que se les diagnosticó DGF con el Cuestionario para Síntomas Gastrointestinales Pediátricos Roma III para Escolares y Adolescentes (QPGS-III), que en estudios previos se ha demostrado su adecuada validez de contenido (10-12) y de constructo (13); y por medio de los Cuestionarios STAIC-1 y STAIC-2, se determinó la presencia de estado-ansiedad y rasgo-ansiedad (5), cuya consistencia interna en niños tiene un alfa de Cronbach entre 0,87 (14) y 0,90 (15). Los niños entre 8 y 10 años hicieron el QPGS-III de manera guiada con uno de los investigadores principales, y los niños entre los 11 y 18 años, por autorreporte.

Se tuvieron en cuenta variables sociodemográficas como edad y sexo, y familiares como padres separados/divorciados, DGF intrafamiliares e hijo único.

Según los Criterios de Roma III se identificaron niños en el grupo de vómito y aerofagia (síndrome de vómito cíclico y síndrome de rumiación del adolescente), de dolor abdominal relacionado a los DGF (dispepsia funcional, síndrome de intestino irritable [SII], migraña abdominal, dolor abdominal funcional [DAF] y síndrome de dolor abdominal funcional [SDAF]) o de estreñimiento e incontinencia (estreñimiento funcional $[\mathrm{EF}]$ e incontinencia fecal no retentiva) (1).

Según el STAIC-1 y el STAIC-2 se identificaron niños con ansiedad transitoria y tendencia a experimentar estados de ansiedad cuando el puntaje total según el sexo fue superior al percentil $50 \%$ (5).

Teniendo en cuenta que se ha reportado una prevalencia de ansiedad en niños con DAR entre el 67,7 \% y $81,6 \%$ (6-9); que se tomaron los niños registrados de la población total de la base de datos del FINDERS $(\mathrm{n}=4394)(16)$; que el valor de z para el nivel de confianza (1-alfa) del $95 \%$ es 1,96 , con una precisión absoluta de 0,05 , y que al tamaño de la muestra se le adicionó un $15 \%$ por no respuesta, el tamaño de muestra total sería de 360 niños; quedando finalmente una sobremuestra de 1496 niños (La Unión, Nariño = 442; Cartagena, Bolívar $=401$, Florencia, Caquetá $=331$ y Santander de Quilichao, Cauca $=322$ ).

Para identificar la posibilidad de errores de transcripción, el $10 \%$ de los datos se revisó y comparó con las formas originales. El análisis estadístico incluyó medidas de tendencia central (promedio, desviación estándar, porcentaje). Para determinar la prevalencia, los datos se analizaron usando una t-student a dos colas, chi cuadrado $\left(\chi^{2}\right)$ y prueba exacta de Fisher. Para las posibles asociaciones se tuvo en cuenta un análisis de regresión con una probabilidad del $20 \%$; reportando un Odds ratio (OR) con sus respectivos intervalos de confianza (IC) del $95 \%$, siendo significativa una $\mathrm{p}<0,05$ (Stata 10 software; StataCorp, College Station, Texas). 


\section{RESULTADOS}

\section{Características generales}

Se incluyeron 1496 escolares y adolescentes de 12,7 $\pm 2,1$ años de edad (rango 8 y 18 años), de los cuales el 50,5\% era de sexo femenino; con diferencias significativas en el grupo de edad de los adolescentes entre los 13 y 18 años de edad; en ser originario de Florencia, Caquetá y Santander de Quilichao, Cauca, y en presentar estado-ansiedad (ansiedad transitoria) y rasgo-ansiedad (tendencia a experimentar estados de ansiedad). El 43,5\% de los niños tenía padres separados/divorciados; el 13,4 \% eran hijos únicos, y el 4,6 \% tenía historia de DGF familiares. En el 18,8\% se identificó algún DGF, presentándose en orden de frecuencia el EF en el 9,2\%; el SII en el 3,7 \%, y el DAF y el SDAF en el 2,3 \% (Tabla 1).

Tabla 1. Características generales de niños colombianos con DGF ( $\mathrm{n}=$ 1496)

\begin{tabular}{|c|c|c|c|}
\hline & \multirow[b]{2}{*}{$\begin{array}{c}\text { Todos } \\
1496\end{array}$} & \multicolumn{2}{|c|}{ DGFs } \\
\hline & & $\begin{array}{c}\text { Si } \\
281(18,8)\end{array}$ & $\begin{array}{c}\text { No } \\
1215(81,2)\end{array}$ \\
\hline Edad (años) & $12,7+/-2,1$ & $12,9+/-2,2$ & $12,7+/-2,1$ \\
\hline $\begin{array}{l}\text { Grupos de edad (n,\%) } \\
\text { - Escolares (8-12 años) } \\
\text { - Adolescentes (13-18 años) }\end{array}$ & $\begin{array}{l}704(47,1) \\
792(52,9)\end{array}$ & $\begin{array}{l}117(41,6) \\
164(58,4)\end{array}$ & $\begin{array}{l}587(48,3) \\
628(51,7)\end{array}$ \\
\hline $\begin{array}{l}\text { Sexo }(n, \%) \\
\text { - Masculino } \\
\text { - Femenino }\end{array}$ & $\begin{array}{l}741(49,5) \\
755(50,5)\end{array}$ & $\begin{array}{l}141(50,2) \\
140(49,8)\end{array}$ & $\begin{array}{l}600(49,4) \\
615(50,6)\end{array}$ \\
\hline $\begin{array}{l}\text { Origen }(\mathrm{n}, \%) \\
\text { - La Unión } \\
\text { - Cartagena } \\
\text { - Florencia } \\
\text { - Santander de Quilichao }\end{array}$ & $\begin{array}{l}442(29,6) \\
401(26,8) \\
331(22,1) \\
322(21,5)\end{array}$ & $\begin{array}{l}81(28,8) \\
83(29,5) \\
86(30,6) \\
31(11,0)\end{array}$ & $\begin{array}{l}361(29,7) \\
318(26,2) \\
245(20,2) \\
291(24,0)\end{array}$ \\
\hline Familiares & & & \\
\hline $\begin{array}{l}\text { DGFs familiares }(n, \%) \\
\text { - No } \\
\text { - Si }\end{array}$ & $\begin{array}{l}1427(95,4) \\
69(4,6)\end{array}$ & $\begin{array}{l}265(94,3) \\
16(5,7)\end{array}$ & $\begin{array}{l}1162(95,6) \\
53(4,4)\end{array}$ \\
\hline $\begin{array}{l}\text { Padres separados/divorciados } \\
(\mathrm{n}, \%) \\
\text { - No } \\
\text { - Si }\end{array}$ & $\begin{array}{l}846(56,5) \\
650(43,5)\end{array}$ & $\begin{array}{l}152(54,1) \\
129(45,9)\end{array}$ & $\begin{array}{l}694(57,1) \\
521(42,9)\end{array}$ \\
\hline $\begin{array}{l}\text { Hijo único }(n, \%) \\
\text { - No } \\
\text { - Si }\end{array}$ & $\begin{array}{l}1296(86,6) \\
200(13,4)\end{array}$ & $\begin{array}{l}245(87,2) \\
36(12,8)\end{array}$ & $\begin{array}{l}1051(86,5) \\
164(13,5)\end{array}$ \\
\hline Ansiedad & & & \\
\hline $\begin{array}{l}\text { Estado }(n, \%) \\
\text { - Si } \\
\text { - No }\end{array}$ & $\begin{array}{l}1195(79,9) \\
301(20,1)\end{array}$ & $\begin{array}{l}236(84,0) \\
45(16,0)\end{array}$ & $\begin{array}{l}959(78,9) \\
256(21,1)\end{array}$ \\
\hline $\begin{array}{l}\text { Rasgo }(n, \%) \\
\text { - Si } \\
\text { - No }\end{array}$ & $\begin{array}{l}770(51,5) \\
726(48,5)\end{array}$ & $\begin{array}{l}159(56,6) \\
122(43,4)\end{array}$ & $\begin{array}{l}611(50,3) \\
604(49,7)\end{array}$ \\
\hline
\end{tabular}

DGF: desórdenes gastrointestinales funcionales

\section{Análisis de asociación}

En el grupo de niños con estado-ansiedad (ansiedad transitoria) hubo predominio en los adolescentes entre los 13 y 18 años de edad (OR: 1,47; IC $95 \%: 1,13-1,91$; p = 0,0026) (Figura 1) y en el sexo masculino (OR: 1,44; IC $95 \%$ : 1,11-1,88; $\mathrm{p}=0,0044$ ) (Figura 2). Al analizar los niños del grupo rasgo-ansiedad (tendencia a experimentar estados de ansiedad), hubo un predominio en los adolescentes (OR: 1,34; IC $95 \%: 1,08-1,65 ; \mathrm{p}=0,0046)$ (Figura 1); en el sexo femenino (OR: 11,84; IC $95 \%$ : 9,22-15,21; p $=0,0000)$ y en los niños con familiares con DGF (OR: 1,94; IC $95 \%$ : 1,13-3,39; $\mathrm{p}=0,0097)$. No se encontraron posibles asociaciones entre la presencia de algún DGF y el estado-ansiedad o rasgo-ansiedad, ni siquiera cuando se analizó individualmente cada uno de los principales DGF (EF, SII, DAF y SDAF) ( $p>0,05)$; ni con las otras variables estudiadas.

Luego de controlar los posibles confusores, las variables que mejor explicaron la presencia de estado-ansiedad (ansiedad transitoria) fueron el grupo etario de los adolescentes y el sexo masculino (Tabla 2); y las que mejor explicaron la presencia de rasgo-ansiedad (tendencia a experimentar estados de ansiedad) fueron el grupo etario de los adolescentes, el sexo femenino y la presencia de algún DGF (Tabla 3).

Tabla 2. Factores asociados con el estado-ansiedad de acuerdo con las variables $(n=1496)$

\begin{tabular}{lccc} 
& \multicolumn{2}{c}{ Estado-ansiedad } & P \\
\cline { 2 - 3 } & OR & IC 95 \% & \\
\hline Adolescentes (13-18 años) & 1,35 & $1,03-1,78$ & 0,027 \\
Sexo masculino & 1,44 & $1,11-1,86$ & 0,005
\end{tabular}

Tabla 3. Factores asociados con rasgo-ansiedad de acuerdo con las variables $(\mathrm{n}=1496)$

\begin{tabular}{lccc} 
& \multicolumn{2}{c}{ Rasgo-ansiedad } & P \\
\cline { 2 - 3 } & OR & IC 95 \% & \\
\hline Adolescentes (13-18 años) & 1,49 & $1,16-1,90$ & 0,001 \\
Sexo femenino & 12,16 & $9,58-15,68$ & 0,000 \\
DGF & 1,43 & $1,05-1,96$ & 0,023
\end{tabular}

\section{DISCUSIÓN}

\section{Prevalencia de los DGF}

En este grupo de escolares y adolescentes colombianos, la prevalencia para presentar algún DGF fue del $18,8 \%$ y el principal DGF fue el EF (9,2\%), seguido del SII $(3,7 \%)$ 


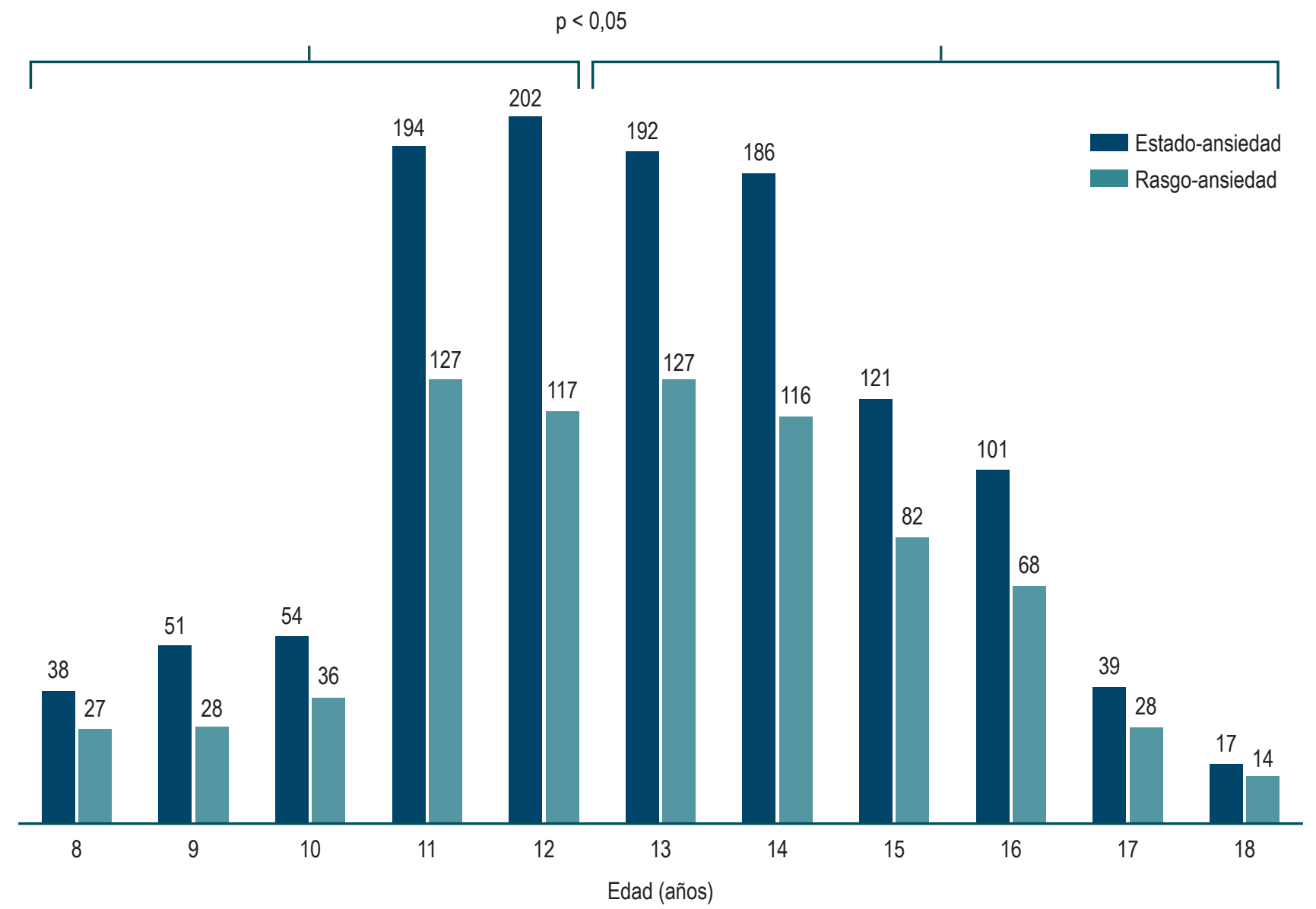

Figura 1. Estado-ansiedad y rasgo-ansiedad según los grupos etarios.

y del DAF junto con el SDAF (2,3\%), entre otros; estos datos son similares a los reportados previamente en Colombia (16), América Latina (4) y el mundo (3).

\section{Prevalencia de estado-ansiedad y rasgo-ansiedad}

La prevalencia para estado-ansiedad y rasgo-ansiedad del presente estudio fue del $20,1 \%$ y $48,5 \%$, respectivamente; datos similares a los reportados por Cunningham y colaboradores (17), quienes en 100 niños norteamericanos con diagnóstico de DAF según los Criterios de Roma III, entre los 8 y 18 años de edad (13,0 $\pm 3,1$ años; 73,0 \% niñas; 87,0 \% caucásicos); $74,0 \%$ con dolor abdominal por más de un año de evolución, a quienes se les identificaron síntomas de ansiedad según el Screen for Child Anxiety Related Disorders-Parent/Child Report (SCARED-C/P), encontraron una prevalencia en los niños con DAF del 54,0 \% de ansiedad (44,0 \% con síntomas pánico-somáticos, $43,0 \%$ con ansiedad generalizada y $42,0 \%$ con ansiedad por separación).

\section{Posibles asociaciones}

En este estudio hubo predominio en el rasgo-ansiedad en los adolescentes, el sexo femenino y poseer DGF intrafamiliares; pero no hubo predominio del estado-ansiedad orasgo-ansiedad para los niños de los grupos con vómito y aerofagia, con dolor abdominal relacionado con los DGF ni con estreñimiento e incontinencia; incluso ni cuando cada DGF fue analizado individualmente como EF, SII, etc. $(p>0,05)$; datos similares a los de Rutten y colaboradores (18), quienes no encontraron diferencias significativas entre los síntomas de ansiedad, el SII y el DAF relacionado con DGF $(p=0,34)$ en 259 niños holandeses entre los 8 y 18 años de edad (132 niños con SII: 59,1\%; SII-e +: $25,8 \%$; SII-m +: 10,6\%; SII-d +: 4,5\%; SII-ss: $13,5 \pm 2,9$ años; $67,4 \%$ niñas y 127 niños con dolor abdominal relacionado con DGF: 40,2 \%; DAF +: 59,8 \%; SDAF: $13,3 \pm 2,7$ años; $74,0 \%$ niñas), a quienes se les determinó ansiedad por medio del Revised Anxiety and Depression Scale - short version (RCADS-25). 


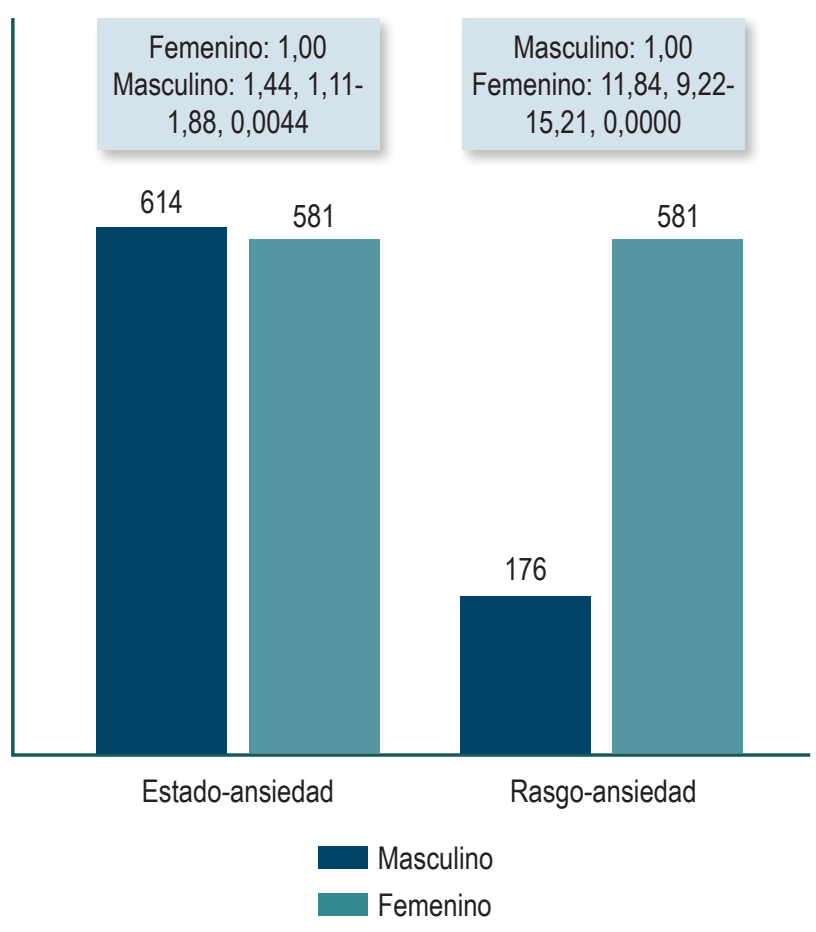

Figura 2. Estado-ansiedad y rasgo-ansiedad según el sexo.

Sin embargo, algunos autores han reportado lo contrario, como Gulewitsch y colaboradores (19), quienes en niños entre los 7,5 y 13 años de edad, 53,3 \% niñas, 18 niños con diagnóstico de DAF y 6 con SII según los Criterios de Roma III con edad promedio de 9,8 $\pm 1,6$ años; 24 niños sanos con $9,9 \pm 1,6$ años y 12 niños con trastornos de ansiedad con 10,1 $\pm 1,6$ años según el STAIC, encontraron que los niños sanos $(32,01 \pm 5,90)$ comparado con los niños con dolor abdominal (DAF + SII $)(38,42 \pm 8,26)$ demostraron mucha más ansiedad según STAIC $(\mathrm{p}<0,01)$; que a su vez no reportaron reacciones alteradas del sistema nervioso parasimpático durante el estrés entre los 3 grupos de estudio.

\section{Posibles factores de riesgo}

El presente estudio encontró posibles factores de riesgo entre el rasgo-ansiedad y el sexo, la edad y la presencia de DGF. En niños norteamericanos entre los 6 y 13 años de edad, 54 niños con diagnóstico de ansiedad (53,7\% niñas, 9,6 $\pm 1,4$ años) y 51 niños sanos (62,7 \% niñas, 9,7 $\pm 1,9$ años) a quienes se les identificaron los síntomas de ansiedad por medio del Spence Children's Anxiety Scale (SCAS-P, SCAS-C) y los trastornos gastrointestinales funcionales por el Questionnaire on Pediatric Gastrointestinal Symptoms (QPGS) Rome Criteria, Waters y colaboradores (20) encontraron que el grupo de niños ansiosos tenía más síntomas de DGF en comparación con el grupo de control ( $p<0,001)$, principalmente con EF; y el grupo de niños ansiosos con DGF presentó mayores puntajes totales al SCAS-C y al SCAS-P en comparación con el grupo de niños ansiosos sin DGF y los del grupo control sanos; así mismo, encontraron una asociación entre la historia familiar de problemas gastrointestinales y el grupo de niños ansiosos con $(\mathrm{p}<0,04)$ y sin DGF $(\mathrm{p}<0,007)$, por lo que se concluyó que la incidencia de síntomas consistentes con DGF fue significativamente mayor en niños con diagnóstico de desórdenes de ansiedad en comparación con los niños no ansiosos.

Pollard y colaboradores (21), en 34 niños norteamericanos $(61,8 \%$ niñas, de $10 \pm 3$ años de edad), encontraron que la disminución de los síntomas gastrointestinales en los pacientes pediátricos con dolor abdominal relacionado con los DGF según los Criterios de Roma III durante los meses de verano está asociado con una disminución de la ansiedad según la Revised Child Anxiety and Depression Scale $(\mathrm{RCAD})$ en este mismo período $(\mathrm{p}=0,017)$; sin embargo, no es claro si la disminución de la ansiedad es la causa o efecto de la disminución de los síntomas del dolor abdominal relacionado con los DGF. Finalmente, Shelby y colaboradores (22), al estudiar 332 niños norteamericanos con dolor abdominal funcional (63,8 \% niñas; $89,7 \%$ blancos; $11,7 \pm 2,5$ años; $40,1 \%$ con DGF, $27,4 \%$ SII + $17,8 \% \mathrm{DF}$ ) y 159 niños de control ( $54,4 \%$ niñas; $94,5 \%$ blancos; 10,8 $\pm 2,0$ años; 7,5 \% con DGF, 6,3 \% SII + 1,9\% DF) y determinar la ansiedad por medio de The Anxiety Disorders Interview Schedule-IV: Adult Lifetime and Child and Parent Versions (ADIS), a quienes por lo menos se hizo seguimiento de 5 años, encontraron una mayor proporción de niños con desórdenes de ansiedad con DAF en comparación con los de control al momento basal (OR: 4,59; IC $95 \%: 2,83-7,43 ; \mathrm{p}=0,001)$ y en el seguimiento (OR: 3,57; IC $95 \%$ : 2,00-6,36; $\mathrm{p}=0,001$ ).

Este estudio fue transversal; sin embargo, estudios longitudinales como el de Cunningham y colaboradores (23), después de realizar seguimiento a 64 niños norteamericanos de 8 a 18 años de edad (promedio de 13,6 años, 42,2 \% con SII; 85,9 \% caucásicos; 75,0 \% niñas) durante 6 meses e identificar posibles factores de riesgo como el dolor, la discapacidad y la ansiedad por medio del Screen for Child Anxiety-related Disorders-Child Report (SCARED-C), encontraron que 2 o más factores de riesgo predicen una evolución tórpida en niños con DGF.

$\mathrm{Al}$ encontrar en este estudio la menor edad como un posible factor protector, y el sexo y la presencia de los DGF como otros factores para desarrollar ansiedad, nuestra hipótesis radica (a excepción de que en el sexo femenino se presente mayor ansiedad) en la variabilidad de los diversos posibles factores predisponentes o de riesgo que epi- 
demiológicamente no han sido confirmados, que incluyen lo genético y constitucional, lo temperamental, los estilos parentales, los acontecimientos vitales estresantes y los ambientes sociales desfavorables.

En conclusión, a pesar de que cerca de la mitad de los niños estudiados presentó una tendencia relativamente estable de tener estados de ansiedad, es difícil saber la magnitud de esta prevalencia, teniendo en cuenta las dificultades metodológicas relacionadas con la delimitación y definición de ansiedad. Así mismo, que se haya reportado un predomino de los niños entre los 13 y 18 años de edad y del sexo femenino, y como posibles factores de riesgo la edad, el sexo y la presencia de los DGF, son necesarios mayores estudios que incluyan otros posibles factores de riesgo desde el punto de vista genético y constitucional, temperamental, de estilo parental, de acontecimientos vitales estresantes y de ambientes sociales desfavorables, entre otros.

\section{Fuente de apoyo financiero}

Ninguna.

\section{REFERENCIAS}

1. Hyams JS, Lorenzo CD, Saps M, Shulman RJ, Staiano A, van Tilgurg M. Childhood Functional Gastrointestinal Disorders: Child/Adolescent. Gastroenterology. 2016;150(5):1456-68. https://doi.org/10.1053/j.gastro.2016.02.015

2. Rasquin A, Di Lorenzo C, Forbes D, Guiraldes E, Hyams JS, Staiano A, Walker LS. Childhood functional gastrointestinal disorders: Child/adolescent. Gastroenterology. 2006; $130(6): 1527-37$. https://doi.org/10.1053/j.gastro.2005.08.063

3. Boronat AC, Ferreira-Maia AP, Matijasevich A, Wang Y-P. Epidemiology of functional gastrointestinal disorders in children and adolescents: A systematic review. World J Gastroenterol. 2017;23(21):3915-27. https://doi.org/10.3748/wjg.v23.i21.3915

4. Velasco-Benítez CA, Saps M, Chanís R, Játiva E, Zablah R, Mejía M, Rodríguez L, Leyva A, Moreno J, Ramírez CR, Sánchez MP, Aragón LE, Nichols-Vinuenza D. La epidemiología de los desórdenes gastrointestinales funcionales en escolares y adolescentes latinoamericanos. Acta Gastroenterol Latinoam. 2017;47(2):148-58.

5. Spielberg CD. State-Trait Anxiety Inventory for Children Professional Manual. Redwood City, CA: Mind Garden, Inc.; 1973.

6. Dufton LM, Dunn MJ, Compas BE. Anxiety and Somatic Complaints in Children with Recurrent Abdominal Pain and Anxiety Disorders. J Pediatr Psychol 2009;34(2):176-86. https://doi.org/10.1093/jpepsy/jsn064

7. Campo JV, Bridge J, Ehmann M, Altman S, Lucas A, Birmaher B, Di Lorenzo C, Iyengar S, Brent DA. Recurrent abdominal pain, anxiety, and depression in primary care. Pediatrics. 2004;113(4):817-24. https://doi.org/10.1542/peds.113.4.817

8. Garber J, Zeman J, Walker L. Recurrent abdominal pain in children: psychiatric diagnoses and parental psychopathology. 1990;29(4):648-56. https://doi.org/10.1097/00004583-199007000-00021
9. Liakopoulou-Kairis M, Alifieraki T, Protagora D, Korpa T, Kondyli K, Dimosthenous E, Christopoulos G, Kovanis T. Recurrent abdominal pain and headache: psychopathology, life events and family functioning. Eur Child Adolesc Psychiatry. 2002;11(3):115-22. https://doi.org/10.1007/s00787-002-0276-0

10. Walker LS, Lipani TA, Greene JW, Caines K, Stutts J, Polk DB, Caplan A, Rasquin-Weber A.. Recurrent Abdominal Pain: Symptom Subtypes Based on the Rome II Criteria for Pediatric Functional Gastrointestinal Disorders. J Pediatr Gastroenterol Nutr. 2004;38(2):187-91.

11. Caplan A, Walker L, Rasquin A. Development and preliminary validation of the questionnaire on pediatric gastrointestinal symptoms to assess functional gastrointestinal disorders in children and adolescents. J Pediatr Gastroenterol Nutr. 2005;41(3):296-304.

12. Caplan A, Walker L, Rasquin A. Validation of the Pediatric Rome II Criteria for Functional Gastrointestinal Disorders Using the Questionnaire on Pediatric Gastrointestinal Symptoms. J Pediatr Gastroenterol Nutr. 2005;41(3):305-16.

13. Saps M, Nichols-Vinueza DX, Mintjens S, Pusatcioglu $\mathrm{CK}$, Velasco-Benítez CA. Construct validity of the pediatric Rome III criteria. J Pediatr Gastroenterol Nutr. 2014;59(5):577-81. https://doi.org/10.1097/MPG.0000000000000482

14. Spielberger CD. Manual for the state-trait inventory for children. Palo Alto, CA: Consulting Psychologists; 1973.

15. Walker LS, Beck JE, Garber J, Lambert W. Children's Somatization Inventory: Psychometric properties of the revised form (CSI-24). J Pediatr Psychol. 2009;34(4):430-40. https://doi.org/10.1093/jpepsy/jsn093

16. Saps M, Moreno-Gómez JE, Ramírez-Hernández CR, Rosen JM, Velasco-Benítez CA. A nationwide study on the prevalence of functional gastrointestinal disorders in school-children. Bol Med Hosp Infant Mex. 2017;74(6):407-12. https://doi.org/10.1016/j.bmhimx.2017.05.005 
17. Cunningham NR, Cohen MB, Farrell MK, Mezoff AG, Lynch-Jordan A, Kashikar-Zuck S. Concordant parentchild reports of anxiety predict impairment in youth with functional abdominal pain. J Pediatr Gastroenterol Nutr. 2013;60(3):312-7. https://doi.org/10.1097/MPG.0000000000000625

18. Rutten JMTM, Benninga MA, Vlieger AM. IBS and FAPS in children: A comparison of psychological and clinical characteristics. J Pediatr Gastroenterol Nutr. 2014;59(4):493-9. https://doi.org/10.1097/MPG.0000000000000452

19. Gulewitsch MD, Weimer K, Enck P, Schwille-Kiuntke J, Hautzinger M, Schlarb AA. Stress reactivity in childhood functional abdominal pain or irritable bowel syndrome. Eur J Pain. 2017;21(1):166-77. https://doi.org/10.1002/ejp.914

20. Waters AM, Schilpzand E, Bell C, Walker LS, Baber K. Functional gastrointestinal symptoms in children with anxiety disorders. J Abnorm Child Psychol. 2013;41(1):151-63. https://doi.org/10.1007/s10802-012-9657-0
21. Pollard KL, Campbell C, Squires M, Palsson O, van Tilburg MAL. Seasonal Association of Pediatric Functional Abdominal Pain Disorders and Anxiety. J Pediatr Gastroenterol Nutr. 2018;67(1):18-22. https://doi.org/10.1097/MPG.0000000000001886

22. Shelby GD, Shirkey KC, Sherman AL, Beck JE, Haman K, Shears AR, Horst SN, Smith CA, Garber J, Walker LS. Functional abdominal pain in childhood and longterm vulnerability to anxiety disorders. Pediatrics. 2013;132(3):475-82. https://doi.org/10.1542/peds.2012-2191

23. Cunningham NR, Jagpal A, Peugh J, Farrell MK, Cohen MB, Mezoff AG, Lynch-Jordan A, Kashikar-Zuck S. Risk categorization predicts disability in pain-associated functional gastrointestinal disorders after 6 months. J Pediatr Gastroenterol Nutr. 2017;64(5):685-90. https://doi.org/10.1097/MPG.0000000000001342 